\title{
Influence of bladder neck suspension stitches on early continence after radical prostatectomy: a prospective randomized study of 180 patients
}

\author{
Jens-Uwe Stolzenburg ${ }^{1, *}$, Martin Nicolaus ${ }^{1, *}$, Panagiotis Kallidonis ${ }^{2}$, Minh Do ${ }^{1}$, Anja Dietel ${ }^{1}$, Tim Häfner ${ }^{1}$, \\ George Sakellaropoulos ${ }^{3}$, James Hicks ${ }^{1}$, David Nikoleishvili ${ }^{1}$ and Evangelos Liatsikos ${ }^{1,2}$
}

Several techniques have been introduced to improve early postoperative continence. In this study, we evaluated the impact of bladder neck (vesicourethral anastomosis) suspension on the outcome of extraperitoneal endoscopic radical prostatectomy (EERPE). In this research, a total of 180 patients underwent EERPE. Group 1 included patients who underwent nerve-sparing EERPE (nsEERPE) $(n=45)$, and Group 2 included patients who underwent nsEERPE with bladder neck suspension (BNS, $n=45$ ). Groups $3(n=45)$ and 4 $(n=45)$ included patients who received EERPE and EERPE with BNS, respectively. Patients were randomly assigned to receive BNS with their nsEERPE or EERPE procedure. Perioperative parameters were recorded, and continence was evaluated by determining the number and weight of absorbent pads (pad weighing test) on the second day after catheter removal and by a questionnaire 3 months postoperatively. Two days after catheter removal, $11.1 \%$ of Group $1,11.1 \%$ of Group $2,4.4 \%$ of Group 3 and $8.9 \%$ of Group 4 were continent. The average urine loss was $80.4,70.1,325.0$ and $291.3 \mathrm{~g}$ for the each of these groups, respectively. At 3 months, $76.5 \%$ of Group 1 and $81.3 \%$ of Group 2 were continent. The continence figures for Group 3 and 4 were $48.5 \%$ and $43.8 \%$, respectively. Similar overall rates were observed in all groups. In conclusion, although there are controversial reports in the literature, early continence was never observed to be significantly higher in the BNS groups when compared with the non-BNS groups, regardless of the EERPE technique performed.

Asian Journal of Andrology (2011) 13, 806-811; doi:10.1038/aja.2011.82; published online 12 September 2011

Keywords: bladder suspension; extraperitoneal endoscopic radical prostatectomy; nerve-sparing; positive surgical margins; prostatectomy; vesicourethral anastomosis

\section{INTRODUCTION}

Endoscopic extraperitoneal radical prostatectomy (EERPE) represents an established method for the treatment of localized prostate cancer. ${ }^{1}$ Increased experience along with the introduction of technical refinements based on recently reported anatomical data have led to improved postoperative functional outcomes with EERPE. ${ }^{1-4}$ However, early continence has been variable among published radical prostatectomy (RP) series, regardless of the approach. ${ }^{5-7}$

Several methods have been proposed to improve early continence after RP. Preservation of the puboprostatic ligaments or bladder neck $(\mathrm{BN})$, placement of pubourethral stitches and suspension of the vesicourethral anastomosis (VUA) to the ligated dorsal venous complex (pubic symphysis) are methods under investigation with controversial results regarding early continence. , $^{2,6-12}$

To elucidate the impact of the bladder neck suspension (BNS) on the outcome of the EERPE procedure, we performed a prospective randomized clinical trial using validated methods for continence evaluation.

\section{MATERIALS AND METHODS}

Patients and inclusion criteria

One hundred and eighty patients underwent EERPE for organconfined prostate cancer between April 2008 and May 2009., ${ }^{1,3}$ The study was approved by the appropriate ethics committees from the institutions. Preoperative evaluation of the participating patients included digital rectal examination, serum prostate-specific antigen (PSA) levels, transrectal biopsy, chest X-ray, pelvic CT and a radioisotope bone scan if the serum PSA was higher than $10 \mathrm{ng} \mathrm{ml}^{-1}$ and/or the Gleason score was higher than 6 .

Four experienced surgeons who had performed more than 300 EERPEs each were involved in the study. These surgeons performed EERPE or bilateral nerve-sparing EERPE (nsEERPE) with or without BNS. The patient population was divided in four groups of 45 patients each based on the EERPE technique used. Group 1 included patients that underwent nsEERPE, and Group 2 included patients that underwent nsEERPE with BNS. Groups 3 and 4 included patients that received EERPE and EERPE with BNS, respectively. All cases were

${ }^{1}$ Department of Urology, University of Leipzig, Leipzig 04103, Germany; ${ }^{2}$ Department of Urology, University of Patras Medical School, Patras 26500 , Greece and ${ }^{3}$ Department of Medical Physics, University of Patras, Patras 26500, Greece

Correspondence: Dr EN Liatsikos (liatsikos@yahoo.com)

Received: 7 February 2011; Revised: 18 April 2011; Accepted: 9 May 2011; Published online: 12 September 2011 
treated consecutively. Patients were initially selected according to whether they were candidates for nsEERPE or EERPE. The patients were then randomized to receive the BNS procedure. The criteria for receiving nsEERPE at our institutions have been previously described in detail. ${ }^{1,3}$ Bilateral nsEERPE was performed in preoperatively potent patients with $\mathrm{T} 1$ or $\mathrm{T} 2$ clinical disease, PSA $<10 \mathrm{mg} \mathrm{ml}^{-1}$ and Gleason score $\leqslant 3+4$. Pelvic lymph node dissection was performed in patients with the following indications: PSA $>10 \mathrm{ng} \mathrm{ml}^{-1}$ and/or Gleason sum $>6$.

\section{EERPE techniques}

The techniques for EERPE and nsEERPE have been described previously in detail. ${ }^{1,3}$ Three sutures suspended the VUA to the posterior of the pubic arch. The first suture was used for the ligation of Santorini's plexus with a 0 Vicryl suture and an $\mathrm{MH}$ plus needle. This suture was placed by selective passage of the needle underneath the plexus from left to right. After placing the knot for plexus ligation, the same suture was passed through the retropubic tissue from right to left, and the urethra was fixed to the posterior pubic symphysis with another knot (Figure 1a and $\mathbf{b}$ ).

The two remaining suspension sutures were placed along with the last two vesicourethral anastomotic stitches. The vesicourethral anastomosis was accomplished during the EERPE procedure by placing eight to nine interrupted sutures. ${ }^{1-3}$ A 2-0 Vicryl suture with a UR-6 needle was used. A variable number of anastomotic sutures were placed at the opening of the BN. All interrupted sutures were placed 'outside-in' at the bladder and 'inside-out' at the urethra and were tied extraluminally. The first suture was placed at the 8:00 o'clock position, and the subsequent four sutures were placed at the 7:00, 6:00, 5:00 and 4:00 o'clock positions. When the dorsal anastomosis was completed, a catheter was inserted to serve as a guide for the rest of the sutures. Systematic placement of sutures at the 3:00, 9:00, 11:00 and 1:00 o'clock positions, which is standard for EERPE procedures, was also performed. The 11:00 and 1:00 o'clock anastomotic sutures also served as suspension sutures and were fixed at the pubic arc slightly lateral to
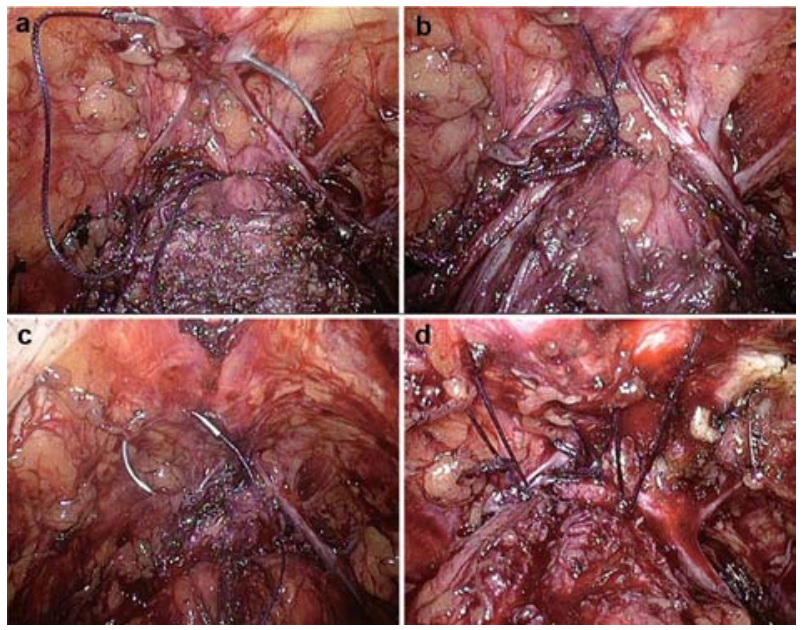

Figure 1 The bladder suspension technique performed during the current series. (a) The first suture was used for ligation of Santorini's plexus (0 Vicryl suture and an MH plus needle) by passing the needle underneath the plexus from left to right. (b) After ligating the plexus, the same suture was passed through the retropubic tissue from right to left, and the urethra was fixed to the posterior pubic symphysis with a knot. (c, d) The 11:00 and 1:00 o'clock anastomotic sutures also served as suspension sutures and were fixed at the pubic arch slightly lateral to the posterior pubic symphysis. the posterior pubic symphysis, as shown in Figure 1c and d. Perioperative parameters were recorded in detail into an electronic database.

\section{Evaluation of continence}

Postoperative continence was evaluated the second day after catheter removal and again at 3 months after EERPE. All patients had at least 3 months of follow-up. Urinary continence was evaluated by the pad weighing test for a standardized bladder volume of $200 \mathrm{ml}$. This procedure is considered the best method for evaluation of postoperative continence. ${ }^{13,14}$ The International Continence Society questionnaire and Expanded Prostate Cancer Index Composite were also used to evaluate continence. ${ }^{15}$ The continence status of the patients was based on their responses to the two following questionnaire items: (i) over the past 4 weeks, how often have you leaked urine; and (ii) How many pads or adult diapers per day did you usually use to control leakage during the last 4 weeks? Continent patients responded that they experienced no leakage of urine and that they used either no pads or one pad for security. Occasional urine leakage requiring two to three pads per day was classified as mild stress incontinence in patients engaged in normal, moderate activity such as walking. Patients requiring more than three pads daily were considered incontinent.

Postoperative cystography was performed in all patients on the fifth postoperative day. The catheter was removed if a watertight anastomosis (i.e., no leakage) was confirmed. All patients received instructions to perform Kegel exercises daily until achieving continence.

\section{Statistical analysis}

For statistical analysis, we compared the perioperative parameters, continence on the seventh day and continence 3 months postoperatively of Groups 1 and 3 with those of Groups 2 and 4, respectively. The Kolmogorov-Smirnov test was used on continuous variables to test possible deviations from the assumption of normality. Variables for which the Kolmogorov-Smirnov test was not significant are presented as mean \pm s.d. in the tables and were assessed by paired $t$-test for significance. For assessments with significant Kolmogorov-Smirnov test values, the variables are presented as 'median (25\%-75\% quartile)' and the Mann-Whitney test (a non-parametric, independent sample median test) was used instead. Comparisons of proportions (e.g., rates of positive margins and lymphadenectomy rate) were assessed by a $z$ test. Discrete variables were analysed by Chi-squared or Fisher's exact tests. Statistical significance was defined as $P<0.05$, and the statistical analysis was processed with WinSTAT version 2007.1 (http:// www.winstat.com).

\section{RESULTS}

The patient demographics and perioperative parameters are summarizsed in Tables $\mathbf{1}$ and $\mathbf{2}$. Mean patient age, PSA values and the number of lymphadenectomies performed were significantly higher in Group 4 compared with Group 3 ( $P=0.019$, Mann-Whitney test).

Pathological results for all groups before and after the procedure are presented in Table 2. Higher postoperative pathological stages $(>\mathrm{T} 2)$ in Groups 3 and 4 are due to the selection criteria used to include patients in the nsEERPE groups. Patients with clinical disease higher than T2 were candidates for 'wide excision' EERPE. Positive surgical margin rates were observed with a similar incidence in all groups.

Continence was assessed by recording pad usage 2 days after catheter removal and again 3 months postoperatively. Tables 3 and 4 present the postoperative continence data for all groups. Two days 
Table 1 Perioperative data ( $n=45$ in each group)

\begin{tabular}{|c|c|c|c|c|c|c|}
\hline & $\begin{array}{c}\text { Group 1 } \\
\text { (nsEERPE) }\end{array}$ & $\begin{array}{c}\text { Group } 2 \\
\text { (nsEERPE+BNS) }\end{array}$ & $\mathrm{P}$ value & Group 3 (EERPE) & $\begin{array}{c}\text { Group } 4 \\
\text { (EERPE+BNS) }\end{array}$ & $\mathrm{P}$ value \\
\hline Age (year) & $61.1 \pm 5.9$ & $59.2 \pm 7.5$ & $0.185^{\mathrm{a}}$ & $65.0 \pm 6.1$ & $67.5 \pm 5.1$ & $0.038^{\mathrm{a}}$ \\
\hline BMI $\left(\mathrm{kg} \mathrm{m}^{-2}\right)$ & $26.4 \pm 3.5$ & $26.0 \pm 2.7$ & $0.530^{\mathrm{a}}$ & $27.6 \pm 3.9$ & $26.2 \pm 3.0$ & $0.074^{a}$ \\
\hline Operative time (min) & $133.6 \pm 32.3$ & $131.6 \pm 24.5$ & $0.738^{\mathrm{a}}$ & $140.9 \pm 25.5$ & $145.2 \pm 29.4$ & $0.465^{a}$ \\
\hline Blood loss (ml) & $252.2 \pm 152.5$ & $248.4 \pm 123.0$ & $0.897^{\mathrm{a}}$ & $200(150-300)$ & $250(150-300)$ & $0.304^{b}$ \\
\hline Transfusion rate (\%) & 0 & 0 & - & 0 & 0 & - \\
\hline Lymphadenectomy performed (\%) & $17.78(8 / 45)$ & $15.56(7 / 45)$ & $0.389^{c}$ & $35.56(16 / 45)$ & $66.67(30 / 45)$ & $0.002^{c}$ \\
\hline
\end{tabular}

Abbreviations: BMI, body mass index; BNS, bladder neck suspension; EERPE, extraperitoneal endoscopic radical prostatectomy; nsEERPE, nerve-sparing EERPE; PSA, prostate-specific antigen.

a Paired $t$-test, variables are presented as mean \pm s.d.

${ }^{\mathrm{b}}$ Mann-Whitney test; variables are presented as median (25\%-75\% quartile).

${ }^{c} z$-test.

Table 2 Pre- and postoperative pathological results

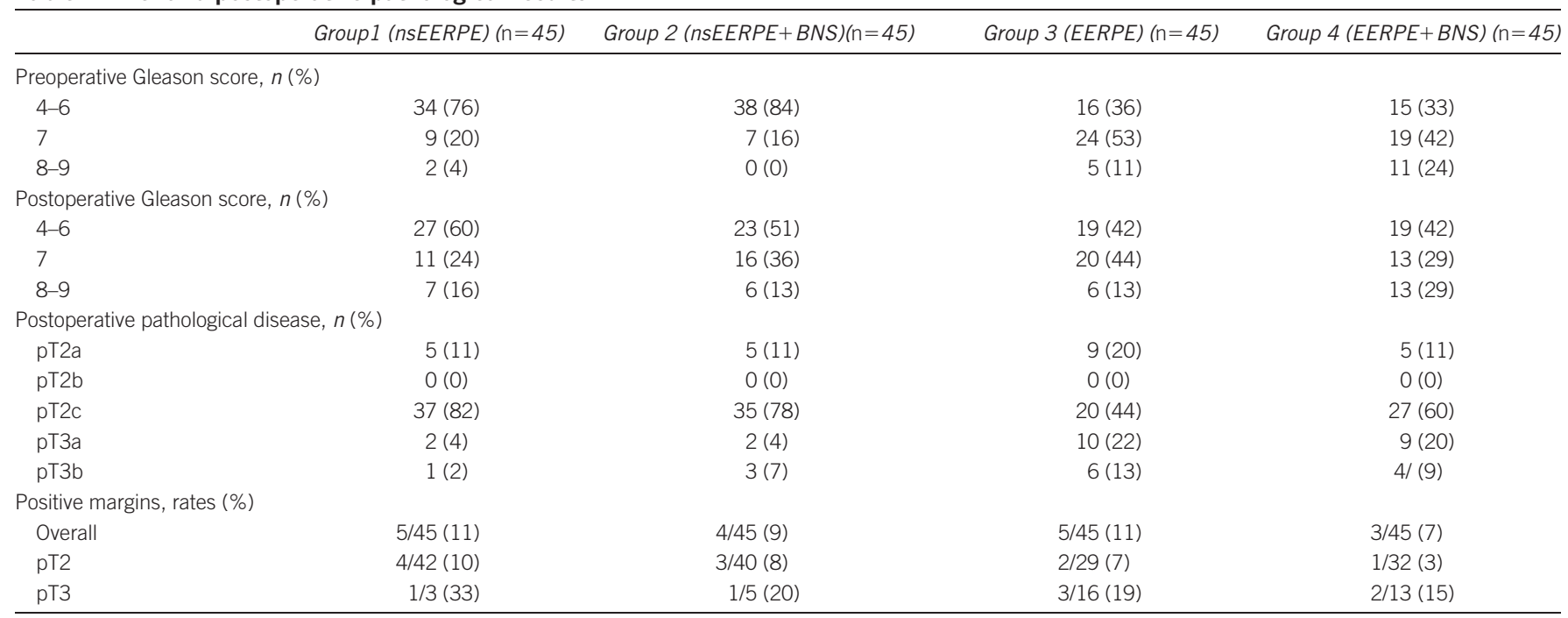

Abbreviations: BNS, bladder neck suspension; EERPE, extraperitoneal endoscopic radical prostatectomy; nsEERPE, nerve-sparing EERPE.

Table 3 Comparison of continence data at 2 days after catheter removal (the seventh postoperative day) and 3 months postoperatively (p.o.) for all groups ( $n=45$ in each group)

\begin{tabular}{|c|c|c|c|c|c|c|}
\hline & $\begin{array}{c}\text { Group1 } \\
\text { (nsEERPE) }\end{array}$ & $\begin{array}{c}\text { Group } 2 \\
\text { (nsEERPE+BNS) }\end{array}$ & $\mathrm{P}$ value & $\begin{array}{l}\text { Group3 } \\
\text { (EERPE) }\end{array}$ & $\begin{array}{c}\text { Group } 4 \\
\text { (EERPE+BNS) }\end{array}$ & $\mathrm{P}$ value \\
\hline No. of pads seventh p.o. day ${ }^{a}$ & $3(2-4)$ & $4(2-5)$ & 0.258 & $6(4-8)$ & $5(3-9)$ & 0.535 \\
\hline Loss of urine (g) seventh p.o. day ${ }^{a}$ & $16.0(6.0-64.0)$ & $27(8-100)$ & 0.383 & $172.0(19.5-631.0)$ & $208.0(14.5-474.5)$ & 0.784 \\
\hline Weight of urine per used pad $(g)^{b}$ & $5.5(2.4-21.1)$ & $8.2(3.0-20.2)$ & 0.505 & $28.8(6.5-60.4)$ & $36.9(3.8-61.0)$ & 0.987 \\
\hline No. of pads used p.o. 3 months & $1(1-1)$ & $1(1-1)$ & 0.200 & $2(1-3)$ & $2(1-2.5)$ & 0.963 \\
\hline
\end{tabular}

Abbreviations: BNS, bladder neck suspension; EERPE, extraperitoneal endoscopic radical prostatectomy; nsEERPE, nerve-sparing EERPE.

Variables are presented as median (25\%-75\% quartile), with $P$ values from Mann-Whitney test.

a PAD test, $24 \mathrm{~h}$.

b PAD test.

after catheter removal, $11.1 \%$ of Group 1, 11.1\% of Group 2, $4.4 \%$ of Group 3 and $8.9 \%$ of Group 4 patients were continent. The average daily pad usages for these groups were $3.5,3.7,6.5$ and 5.9, respectively. The average urine losses per day were $80.4 \mathrm{~g}$ and $70.1 \mathrm{~g}$ for the Group 1 and Group 2 nsEERPE patients, respectively. In Groups 3 and 4, the average loss of urine was $325.0 \mathrm{~g}$ and $291.3 \mathrm{~g}$, respectively. After 3 months, $76.5 \%$ of Group 1 and $81.3 \%$ in Group 2 were continent. The respective continence figures for Groups 3 and 4 were $48.5 \%$ and $43.8 \%$ after 3 months, respectively. BNS did not contribute to early continence in patients who underwent nsEERPE or EERPE.

Due to the positive skew of the observed data, power calculations were made without the assumption of normal distribution. Therefore, we performed the Mann-Whitney test for non-inferiority, assuming that the actual distribution was double exponential. ${ }^{16-18}$ 
Table 4 Continence of patients ( $n=45$ in each group)

\begin{tabular}{|c|c|c|c|c|c|c|}
\hline No. of pads per day & Group 1 & Group 2 & $\mathrm{P}$ value & Group 3 & Group 4 & P value \\
\hline \multicolumn{7}{|c|}{ Two days after catheter removal } \\
\hline $0-1$ & $5 / 45(11.1)$ & $5 / 45(11.1)$ & 0.202 & 2/45 (4.4) & $4 / 45(8.9)$ & 0.696 \\
\hline $2-3$ & $24 / 45(53.3)$ & $16 / 45(35.6)$ & & $8 / 45(17.8)$ & $8 / 45(17.8)$ & \\
\hline$>3$ & $16 / 45(35.6)$ & 24/45 (53.3) & & $35 / 45(77.8)$ & $33 / 45(73.3)$ & \\
\hline \multicolumn{7}{|l|}{ Postoperative 3 months } \\
\hline $0-1$ & 26/34 (76.5) & 26/32 (81.3) & 0.579 & $16 / 33(48.5)$ & $14 / 32(43.8)$ & 0.901 \\
\hline $2-3$ & $7 / 34(20.6)$ & $4 / 32(12.5)$ & & 12/33 (36.4) & 12/32 (37.5) & \\
\hline$>3$ & $1 / 34(2.9)$ & $2 / 32(6.3)$ & & 5/33 (15.2) & $6 / 32(18.8)$ & \\
\hline
\end{tabular}

Data are presented as patient number rate (\%). $P$ values are for Chi-squared test.

Power was calculated by setting the margin of equivalence to 34.0 , which is equal to the difference between the median (16.0) of the standard group (nsEERPE) and the value that indicates the limit for severe incontinence according to the International Continence Society recommendations (50.0). ${ }^{19}$ The true difference between the means was assumed to be -10.0 , which is equal to the observed difference in means between the groups. The observed standard deviations of Groups 1 and 2 were used for the calculations (155.7 and 106.4, respectively). The significance level (alpha) of the test was set to 0.05 . With sample sizes of 45 per group, we achieved $60 \%$ power to detect non-inferiority of the nsEERPE+BNS procedure when compared with standard nsEERPE. For Groups 3 and 4, the margin of equivalence was set to 122.0, which is equal to the difference between the median (172.0) of the standard group (EERPE) and the limit for severe incontinence (50.0). The true difference between the means was assumed to be -33.7 , which is equal to the observed difference in the means of Groups 3 and 4. The observed standard deviations of Groups 3 and 4 used for the calculations were 371.3 and 319.9, respectively. With sample sizes of 45 per group, we achieved $82.7 \%$ power to detect non-inferiority of the EERPE+BNS procedure when compared to standard EERPE.

\section{DISCUSSION}

Mechanisms for male pre- and postprostatectomy urinary continence are presented in the literature; however, there is no definite conclusion among the investigators. Damage to the pelvic floor and/or external urethral sphincter musculature and loss of anterior urethral support have been associated with varying degrees of postoperative incontinence. Moreover, age needs to be considered as a predisposing factor for incontinence. $^{2}$ The precise anatomical structure of the ligaments and suspension structures of the $\mathrm{BN}$ and their effect on continence are still unclear in men. In contrast, the compartment theory of the pelvic floor in females has been developed and adapted by pelvic surgeons. ${ }^{20}$

Radical prostatectomy techniques have achieved postoperative continence during the first postoperative year. Recently, urologic research has also focused on the improvement of early postoperative continence. ${ }^{6}$ However, the improvement of continence after catheter removal (approximately after the first postoperative week) and again after 3 months is a challenge for surgical techniques.

Different techniques have been proposed to improve post-prostatectomy continence, with varying results. Preservation of the puboprostatic ligaments has contributed to early continence after prostatectomy. ${ }^{2,7}$ Klein $^{21}$ was the first to report technical modifications to preserve the $\mathrm{BN}$ and to improve continence. Several other investigators have concentrated on $\mathrm{BN}$ preservation as a method to improve postoperative continence, with controversial results regarding the contribution of the technique to both postoperative contin-

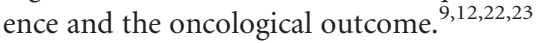

Urethral and BN suspension by various methods (e.g., stitches and slings) has been used in the management of stress incontinence for several years. ${ }^{24}$ Walsh and Partin ${ }^{25}$ described a technical refinement that divided the dorsal venous complex (DVC) with minimal blood loss while taking care not to injure the striated sphincter. In this technique, a suture is passed through the DVC and through the posterior of the pubic symphysis, and then the same manoeuvre is performed in the reverse direction. The VUA is suspended towards the pubic bone, and the provided recapitulation of the puboprostatic ligaments supports the striated sphincter when the suture is tied.

Noguchi et al. ${ }^{26,27}$ introduced a suspension manoeuvre for the VUA during radical retropubic prostatectomy (RRP). These investigators used anastomotic sutures at the 1:00 and 11:00 o'clock positions to anchor the VUA to the ligated DVC, which results in suspension of the anastomosis. They observed that the suspension technique provided significant improvement in continence 1,3 and 6 months postoperatively. The respective continence rates for the above time periods were $53 \%, 73 \%$, and $100 \%$ for the bladder suspension group and $20 \%, 47 \%$ and $83 \%$ for the control group. Based on these results, the authors concluded that bladder suspension significantly contributes to early continence recovery and proposed technical modifications for other RP approaches.

Campenni et al. ${ }^{28}$ performed VUA suspension by placing two pubourethral suspension stitches during RRP. This method does not interfere with the anatomy or introduce a foreign body (sling). The suspension stitches are placed in the anterior VUA and anchored to the retropubis and periostium. In total, $32 \%$ of patients who underwent bladder suspension were continent at 6 months, whereas only $12 \%$ of the control group were continent in the same time frame. The investigators suggested that urethral suspension may increase the incidence of early postoperative continence.

Recently, Patel et al. ${ }^{6}$ described a technique for placing a periurethral suspension stitch during robotic-assisted radical prostatectomy in detail. The stitch is inserted between the DVC and the urethra and passed on the pubic bone. The stitch is again passed between the urethra, DVC and pubic bone in a figure-eight fashion. Significantly improved continence was observed in the periurethral suspension group compared with the non-suspension group 3 months postoperatively.

These techniques that focus on BNS and postoperative early continence have several limitations that should be taken into consideration when interpreting the results. The majority of these studies were prospective studies, but they were not always randomized. ${ }^{6,28}$ All studies evaluated continence 3 months postoperatively, ${ }^{6,26-28}$ and Noguchi et al. ${ }^{26}$ performed continence evaluation as early as 1 month after RP. Thus, early postoperative continence evaluation after catheter removal was never assessed. Because the pad test is considered the most reliable incontinence assessment tool and has already been used for predicting continence outcome after radical prostatectomy, early continence 
after BNS procedures should be evaluated accordingly. ${ }^{29-31}$ All of the aforementioned BNS studies evaluated continence by questionnaires, which are a less reliable method for continence assessment when compared with the pad test. ${ }^{14,29}$ In the present study, we assessed early continence using pad weighing tests as well as questionnaires.

Another limitation of the current literature is the lack of stratified outcome data concerning BNS and postoperative early continence in light of technical refinements, such as the nerve-sparing technique. ${ }^{6,26-28}$ In our study, we evaluated the BNS technique in both EERPE and nsEERPE and assessed the influence of the EERPE technique on early postoperative continence. Patients were selected for EERPE or nsEERPE procedures according to specific criteria and were prospectively randomized to receive bladder suspension. All surgeons had overcome their learning curve so that technical competence did not influence the outcome of the EERPE. Perioperative data showed that the patients in Group 4 underwent a significantly higher number of lymph node dissections, which is likely associated with the significantly higher PSA values observed in this same group when compared with Group 3. Moreover, the EERPE groups included more patients with Gleason scores higher than 7 due to the inclusion criteria for EERPE. The extent of periprostatic fascia preservation and the performance of a nerve-sparing procedure have been positively associated with improved postoperative continence. ${ }^{32}$ The nsEERPE continence outcome was improved in comparison to EERPE in our study (Tables 3 and 4).

Pelvic lymph node dissection was performed in $35.56 \%$ and $66.67 \%$ of patients in Groups 3 and 4, respectively. In contrast, pelvic lymph node dissection was performed in $17.78 \%$ and $15.56 \%$ of patients in Groups 1 and 2, respectively. The difference in lymph node dissection rates reflects the inclusion criteria for lymphadenectomy because patients with lower staging were placed in the nsEERPE groups. The positive surgical margin rates for the groups in this study were similar to each other and were also in agreement with the values reported in the literature. $1,7,33$

Another limitation of this study is the lack of data regarding patient's quality of life (QoL). It is possible that QoL indicators were significantly impacted by incontinence differences of $10 \%-20 \%$. Incontinence could also be assessed according to the International Continence Society classification for the pad weighing test. ${ }^{19}$ However, the comparative assessment presented in this study provides a better depiction of the relationship between BNS and continence. Therefore, the comparative assessment provides sufficient information regarding continence results, but the impact of the continence rates on quality of life parameters remains unclear. QoL assessments may have been helpful to our study, and the sample size needed to assess QoL between the different groups would have been taken into account if this assessment was included. While we recognize this limitation, we believe that both the power calculation methodology and the specific power of our study with regard to the reported differences are appropriate and are included in detail in this manuscript.

Patel et al. ${ }^{6}$ noted significant improvement in the BNS group 3 months postoperatively. However, these investigators used only validated questionnaires to document continence. The use of validated continence evaluation questionnaires in conjunction with measuring both the number of pads used daily and the weight of the pads lends additional integrity to the results of the present study. ${ }^{30}$

\section{CONCLUSION}

VUA suspension with stitches at the posterior pubic symphysis did not improve early postoperative continence in patients undergoing
EERPE, regardless of the performance of nerve-sparing techniques. Further clinical evaluation of techniques that may provide early continence after RP is deemed necessary.

\section{AUTHOR CONTRIBUTIONS}

J-US designed the study and interpreted the data. MN designed the study. PK interpreted the data, conducted statistical analysis, drafted and revised the manuscript, $\mathrm{MD}, \mathrm{AD}$ and $\mathrm{TH}$ conducted the data acquisition. GS conducted statistical analysis. JH conducted data interpretation and manuscript drafting. DN interpreted data, and EL designed the study and interpreted the data.

\section{COMPETING FINANCIAL INTERESTS}

The authors declare no competing financial interests.

1 Stolzenburg JU, Kallidonis P, Minh D, Dietel A, Häfner T et al. Endoscopic extraperitoneal radical prostatectomy: evolution of the technique and experience with 2400 cases. J Endourol 2009; 23: 1467-72.

2 Stolzenburg JU, Liatsikos EN, Rabenalt R, Do M, Sakelaropoulos G et al. Nerve sparing endoscopic extraperitoneal radical prostatectomy — effect of puboprostatic ligament preservation on early continence and positive surgical margins. Eur Urol 2006; 49: 103-12.

3 Stolzenburg JU, Rabenalt R, Do M, Schwalenberg T, Winkler M et al. Intrafascial nerve-sparing endoscopic extraperitoneal radical prostatectomy. Eur Urol 2008; 53: 931-40.

4 Liatsikos E, Assimakopoulos K, Stolzenburg JU. Quality of life after radical prostatectomy. Urol Int 2008; 80: 226-30.

5 Bauer RM, Bastian PJ, Gozzi C, Stief CG. Postoprostatectomy incontinence: all about diagnosis and management. Eur Urol 2009; 55: 322-33.

6 Patel VR, Coehlo RF, Palmer KJ, Rocco B. Periurethral suspension stitch during robotic-assisted laparoscopic radical prostatectomy: description of the technique and continence outcomes. Eur Urol 2009; 56: 472-8.

7 Ficcara V, Novara G, Artibani W, Cestari A, Galfano A et al. Retropubic, laparoscopic and robot-assisted radical prostatectomy: a systematic review and cumulative analysis of comparative studies. Eur Urol 2009; 55: 1037-63.

8 Lowe BA. Preservation of the anterior urethral ligamentous attachments in maintaining post-prostatectomy urinary continence: a comparative study. J Urol 1997; 158: 2137-41.

9 Deliveliotis C, Protogerou V, Alargof E, Varkarakis J. Radical prostatectomy: bladder neck preservation and puboprostatic ligament sparing-effects on continence and positive margins. Urology 2002; 60: 855-8.

10 Selli C, de Antoni P, Moro U, Macchiarella A, Giannarini G et al. Role of bladder neck preservation in urinary continence following radical retropubic prostatectomy. Scand J Urol Nephrol 2004; 38: 32-7.

11 Walsh PC, Marschke PL. Intussusception of the reconstructed bladder neck leads to earlier continence after radical prostatectomy. Urology 2002; 59: 934-8.

12 Srougi M, Nesrallah LJ, Kauffman JR, Nesrallah A, Leite KR. Urinary continence and pathological outcome after bladder neck preservation during radical retropubic prostatectomy: a randomized prospective trial. J Urol 2001; 165: 815-23.

13 Lose G, Rosenkilde P, Gammelgaard J, Schroeder T. Pad-weighing test performed with standardized bladder volume. Urology 1988; 32: 78-80.

14 Siltberg H, Victor A, Larsson G. Pad weighing tests: the best way to quantify urine loss in patients with incontinenceActa Obstet Gynecol Scand Supp/ 1997;166: 28-32.

15 Wei J, Dunn R, Litwin M, Sandler H, Sanda M. Development and validation of the Expanded Prostate Cancer Index Composite (EPIC) for comprehensive assessment of health-related quality of life in men with prostate cancer. Urology 2000; 56: 899905.

16 Al-Sunduqchi MS. Determining the appropriate sample size for inferences based on the Wilcoxon statistics. PhD dissertation under the direction of William C. Guenther, University of Wyoming, Laramie, WY, 1990.

17 Chow SC, Shao J, Wang H. Sample Size Calculations in Clinical Research. New York: Marcel Dekker; 2003.

18 Julious SA. Sample sizes for clinical trials with normal data. Stat Med 2004; 23 : 1921-86.

19 Smither AR, Guralnick ML, Davis NB, See WA. Quantifying the natural history of post-radical prostatectomy incontinence using objective pad test dataBMC Urol 2007; 7: 2.

20 Fritsch H, Lienemann A, Brenner E, Ludwikowski B. Clinical Anatomy of the Pelvic Floor. Advances in Anatomy and Cell Biology. Vol. 175. Berlin/Heidelberg: SpringerVerlag; 2004.

21 Klein EA. Early continence after radical prostatectomy. J Urol 1992; 4892-5. 
22 Shelfo SW, Obek C, Soloway MS. Update on bladder neck preservation during radical retropubic prostatectomy: impact on pathologic outcome, anastomotic strictures, and continence.Urology1998;51: 73.

23 Poon M, Ruckle H, Bamshad BR, Tsai C, Webster R et al. Radical retropubic prostatectomy: bladder neck preservation versus reconstruction.J Urol 2000; 163: 194.

24 Barbalias G, Liatsikos E, Barbalias D. Use of slings made of indigenous and allogenic material (Goretex) in type III urinary incontinence and comparison between them. Eur Urol 1997; 31: 394-400.

25 Walsh PC. Anatomic radical retropubic prostatectomy. In: Walsh PC, Retnik AB, Wein AJ, editors. Campbell's Urology. 8th ed. Philadelphia, PA: Saunders; 2002: 3107-26.

26 Noguchi M, Shimada A, Nakashima O, Kojiro M, Matsuoka K. Urodynamic evaluation of a suspension technique for rapid recovery of continence after radical retropubic prostatectomy. Int J Urol 2006; 13: 371-6.

27 Noguchi M, Kakuma T, Suekane S, Nakashima O, Mohamed ER et al. A randomized clinical trial of suspension technique for improving early recovery of urinary continence after radical retropubic prostatectomy. BJU Int 2008; 102: 958-63.
28 Campenni MA, Harmon JD, Ginsberg PC, Harkaway RC. Improved continence after radical retropubic prostatectomy using two pubourethral suspension stitches. Urol Int 2002; 68: 109-12.

29 Franco AV, Lee F, Fynes MM. Is there an alternative to pad test? Correlation of subjective variables of severity of urinary loss to the 1-h pad test in women with urinary stress incontinence. BJU Int 2008; 102: 586-9.

30 Ates M, Teber D, Gozen AS, Tefleki A, Hruza M et al. A new postoperative predictor of time of continence after laparoscopic radical prostatectomy. Eur Urol 2007; 52: 178-85.

31 van Kampen M, Geraerts I, de Weerdt W, van Poppel H. Easy predictor of urinary incontinence after retropubic radical prostatectomy based on urinary loss the first day after catheter withdrawal. J Urol 2009; 181: 2641-6.

32 van der Poel HG, de Blok W, Joshi N, van Muilekom E. Preservation of lateral prostatic fascia is associated with urine continence after robotic-assisted prostatectomy. Eur Urol 2009; 55: 892-901.

33 Toujier K, Secin FP, Cronin AM, Katz D, Bianco F et al. Oncologic outcome after laparoscopic radical prostastectomy: 10 years of experience. Eur Urol 2009; 55: 1014-9. 